\title{
Practical and User-Friendly Circuits and System Design for Signals' Sensing and Generation
}

\author{
Ching-Hwa $\mathrm{Ho}^{{ }^{*}}$, Ji-Hsien $\mathrm{Ho}^{2}$ \\ ${ }^{1}$ Graduate Institute of Applied Science and Technology, National Taiwan University \\ of Science and Technology, Taipei, Taiwan \\ ${ }^{2}$ Media and Visual Communication, Department of Industrial Design, Chang Gung University, \\ Tao-Yaun, Taiwan \\ Email: ${ }^{*}$ chho@mail.ntust.edu.tw
}

Received July 6, 2013; revised August 6, 2013; accepted August 13, 2013

Copyright (C) 2013 Ching-Hwa Ho, Ji-Hsien Ho. This is an open access article distributed under the Creative Commons Attribution License, which permits unrestricted use, distribution, and reproduction in any medium, provided the original work is properly cited.

\begin{abstract}
Design and implementation of a personal computer (PC) based measurement circuits \& system that containing signals' sensing and generation are demonstrated in this study. The instrument can be easily operated via a user-friendly interface consisted of some functional keys displayed on the PC screen. Compact design of the hardware for the two units (signals' sensing and generation) is made in a plug-in style of PC input/output (I/O) card so that no extra space for the instrument is needed. Design concepts for the hardware and software of the instrument are described. Functional performances of the setup of signals' sensing and generation are tested. The results show user-friendly function and wellbehaved performance for the package design.
\end{abstract}

Keywords: A/D Conversion; D/A Conversion; Data Acquisition; I/O Interface Cards; Auto-Testing Equipment

\section{Introduction}

The apparatuses of signals' sensing (SS) and generation (SG) are important equipments for testing analog and digital signals in laboratory. The electrical engineers usually need a good sensing and monitor tool to analyze electrical signals they want to measure. A practical sensing and monitor tool must support many useful functions such as data storage, computer linking, numeric analysis, and curve printing to meet different demands by users. To match the fundamental requirements, a SS device must be computerized because numeric data processing and data storage are the essential functions for users. Furthermore, the experimentalists in laboratory usually require a proper $\mathrm{SG}$ device to generate some specific functional signals for testing. A practical SG device should generate not only the basic functions of sine, triangle, and square waves but also the signal with a userdefined waveform. To generate user-defined waveforms, a fully computerized SG is necessary to be developed. From the experimentalists' point of view, a fully computerized auto-testing-equipment (ATE) that combining both SS and SG apparatuses should be essential in fulfilling their experimental testing tasks.

*Corresponding author.
A personal computer (PC) based ATE is now a very convenient instrumentation system. The PC-based SS device had ever been utilized for studying nonvolatile memories [1], for simulating virtual spectrum analyzer, imitating digital image processor $[2,3]$, and checking the function of high-frequency power electronics [4]. The functions of PC-controlled SG can be a waveform generator [1], a programmable constant current source to generate electroluminescences from optoelectronic devices [5], and for generation of timing clocks to drive linear charge-coupled-device (CCD) arrays [5,6]. Although the individual SS or SG device had ever been found, a systematic study on overall understanding of the actual design and implementation of electronic hardware and software of a PC auxiliary ATE would be valuable to be announced herein.

In this paper, practical design and implementation of a real ATE measurement package are demonstrated (i.e. system diagram as shown in Figure 1). The instrument is easily operated via some user-friendly functional keys displayed on the PC screen. The hardware for the SS and SG is fabricated in a plug-in style of PC I/O card so that no extra instrumentation space is needed. The sampling frequency of the SS card can be programmably setting by user. The maximum sampling rate is up to $19 \mathrm{MHz}$. The 


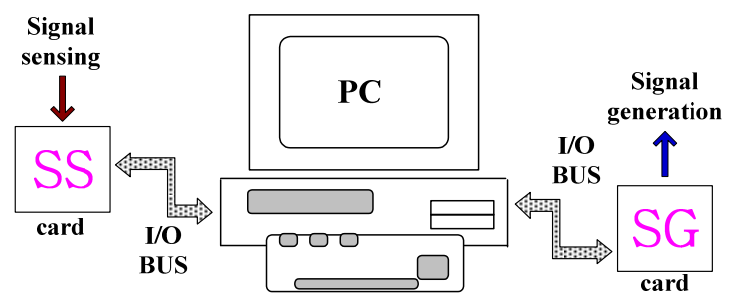

Figure 1. System diagram for the PC-based measurement package of signals' sensing and generation.

output signal of the SG device can be user-defined by plotting the waveform on the PC screen and then sending it out periodically via the SG card. Design diagrams of the hardware and software of the ATE system are described. Some experimental examples for demonstrating the functional behavior of both SS and SG devices are exhibited. The experimental results show well-behaved performance of the package design.

\section{Instrument Design}

\subsection{Design and Implementation of PC-Based Signals' Sensing Device}

The electronic-circuits design for the SS interface card is illustrated in Figure 2. The SS device possesses two input channels denoted as $\mathrm{CH} 1$ and $\mathrm{CH} 2$. The input part for each channel consists of a capacitor $(25 \mathrm{pF})$ and an AD844 based unit-gain inverter [7]. The AD844 is a high-speed operational amplifier (OP) which possesses a wide bandwidth of $60 \mathrm{MHz}$ and a high slew rate of 2000 $\mathrm{V} / \mu \mathrm{s}$. The input resistor for the unit-gain inverter is 1 $\mathrm{M} \Omega$ so that the input impedance for the SS device is similar to the general oscilloscope of $\mathrm{C}_{\mathrm{in}}=25 \mathrm{pF}$ and $\mathrm{R}_{\text {in }}$ $=1 \mathrm{M} \Omega$. As shown in Figure 2, in connection with the unit-gain inverter, another inverting amplifier based on an AD844 OP is found. The inverting amplifier consists of an analog switch DG508 in combination with some resisters of $1 \mathrm{k}, 10 \mathrm{k}, 100 \mathrm{k}, 1 \mathrm{M}$, and $10 \mathrm{M} \Omega$. The components are used to determine the amplification gain by selecting the resistors via DG508. By properly setting the gain, a suitable level of input signal can be applied to the main analog-to-digital (A/D) converter denoted as AD9058 [8]. The AD9058 contains two independent 8-bits A/D channels on a monolithic chip. Both A/D channels possess a fast conversion rate of 40-mega samples per second (40 MSPS). To prevent the AD9058 from overshot damage, a clipper circuit that consisted of four silicon diodes is utilized for limitation of the signal amplitude to within $\pm 1.4 \mathrm{~V}_{\mathrm{pp}}$. The reference voltage of $+\mathrm{V}_{\mathrm{REF}}\left(-\mathrm{V}_{\mathrm{REF}}\right)$ for $\mathrm{AD} 9058$ is set at $+1.5 \mathrm{~V}(-1.5 \mathrm{~V})$ by an adjustable voltage regulator consisted of an AD844 and an n-p-n (p-n-p) transistor. The sampling clocks for the $\mathrm{A} / \mathrm{D}$ conversion are originated from a $38 \mathrm{MHz}$ crystal oscillator and then programmed by a programmable counter denoted as $82 \mathrm{C} 54$. The sampling rate for the $\mathrm{A} / \mathrm{D}$ conversion can be set from a low frequency up to a maximum value of $\sim 19 \mathrm{MHz}$. The 8-bits digital data converted from each A/D channel of AD9058 are sent to a tri-state latch $74 \mathrm{~F} 373$, and then stored in a memory buffer 6116. An 11-bits ripple counter using 74LS93 is utilized for addressing 2 kilo-bytes data in the 6116 memory buffer. The data storage in the memory buffer is accomplished by simultaneously sending out the sampling clocks to AD9058 and the 11-bits addressing counter, and then turned on the tri-state latch, and finally stored the converted digital data into 6116. Two 8255A programmable-peripheral-interface (PPI) chips handle the data communication between PC and the SS card. The programming control of data acquisition of the SS device is accomplished by setting the amplification gain and choosing the sampling frequency, and then storing the converted digital data into a memory buffer. When PC reads out the stored data from the memory buffer, the waveform of the measured signal can be depicted on the PC screen. The prototype for the electronic hardware of the SS card is now fabricated in a plug-in style of PC I/O card. If we replace the electronic parts with surface mounting components of compact size the dimension of the SS card can be properly reduced.

Figure 3 shows the user-friendly operation interface for the SS device. It is designed and programmed using $\mathrm{C}$ language. The waveform data derived from $\mathrm{CH} 1$ and $\mathrm{CH} 2$ (in the SS card) can be simultaneously displayed on the SS monitor in Figure 3. The operation interface of the SS monitor is user friendly, which can execute different tasks of the SS by using only mouse click on the functional keys. The basic function keys include the setting of sampling frequency, selection of volt division, data smoothing, data printing, and data recalling, etc.

\subsection{Electronics Design of the Signals Generator}

The circuits' design of the electronic hardware of the SG card is illustrated in Figure 4. A $82 \mathrm{C} 54$ is utilized for setting the frequency of the output waveform derived from PC. A 256-bytes memory buffer using 6116 is utilized for storage of the digital data of output waveform. An 8-bits ripple counter formed by two cascade 74LS93 is adopted for addressing the 256-bytes memory buffer. The digital-to-analog (D/A) conversion for the generation of functional waveform is accomplished by using 8-bits D/A converter DAC0808. DAC0808 is a current-mode $\mathrm{D} / \mathrm{A}$ converter which converts 8 -bits digital data to the corresponding value of output current. The output error is about one least significant bit of the 8-bits of $\sim \pm(5 \mathrm{~V} / 5 \mathrm{k} \Omega) / 255= \pm 3.92 \mu \mathrm{A}$ depending on the circuit's design. The output current can be converted into output voltage by a current-to-voltage (I to $\mathrm{V}$ ) converter using AD844. The operation of the SG device is accom- 




Figure 2. Circuits design for the PC-based signals' sensing card.

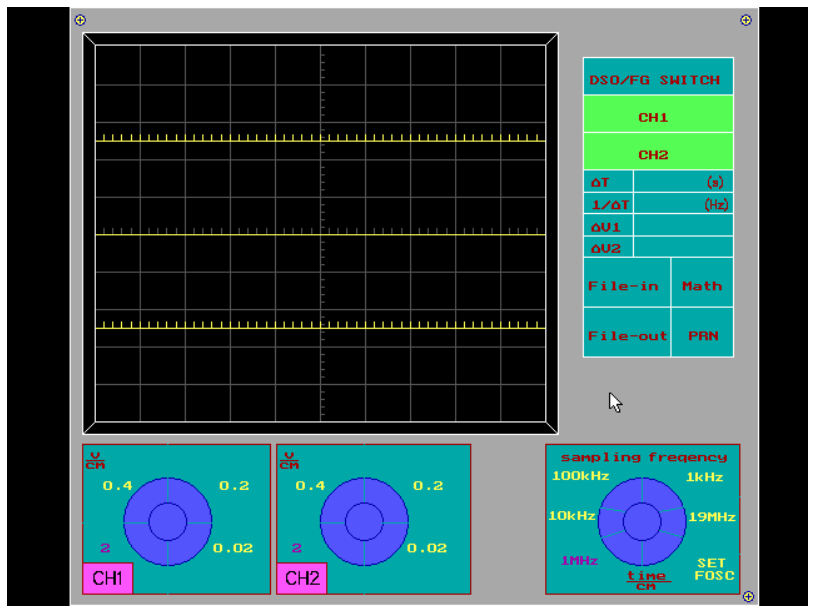

Figure 3. The user-friendly interface for operation of the SS device on the PC screen.

plished by drawing a curve in the SG monitor and then stores the corresponding digital data into the 256-bytes memory buffer via I/O control of $8255 \mathrm{~A}$. The frequency of output signal is determined by setting the $82 \mathrm{C} 54$ programmable interval counter. By sending out the digital data from 6116 to the D/A converter successively, a stream of analog signals can be periodically generated to the output terminal of the SG.

The controlled panel for the PC programmable SG device is illustrated in Figure 5. The SG device generates not only the signals of sine, square, and triangular waves but also the signal with user-defined waveform. The signal generation is achieved by clicking the functional keys of sine, square, or triangular wave on the SG monitor. Figure 5 shows a sine curve produced by clicking the sine-wave key on the SG monitor. After setting the frequency and amplitude of the waveform, the output signal can be generated. For creation of an arbitrary waveform, a user-defined shape should be sketched on the PC screen and the values of frequency and amplitude need to set. After the parameters' setting, corresponding digital data will be calculated and sent out to the SG card for production of an analog signal.

\section{Experimental and Testing Results}

The experimental result for testing the performance of the dual-channels SS device is shown in Figure 6. The signal source is a commercialized function generator. CH1 displays a triangular wave with a frequency of 790 


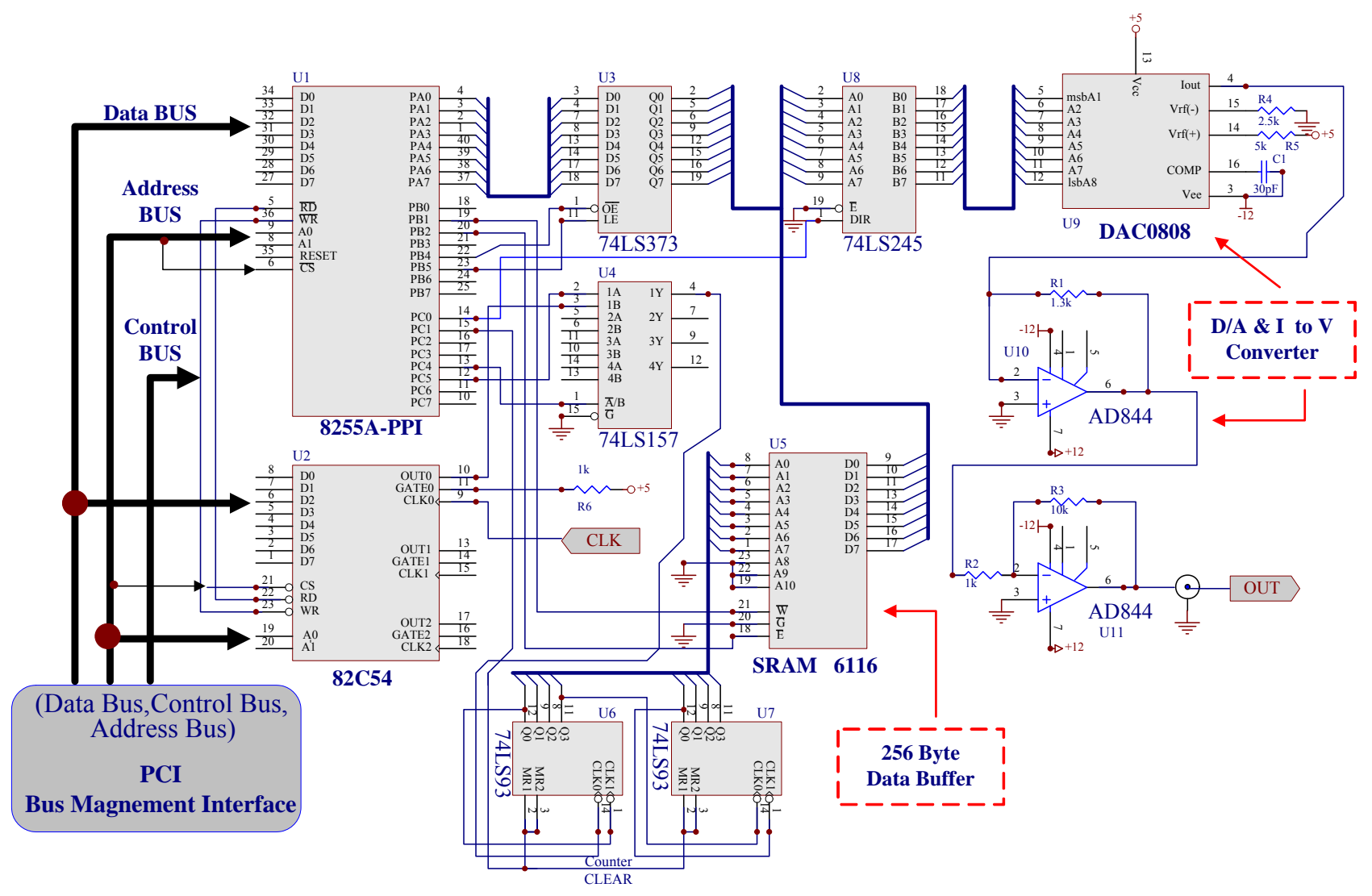

Figure 4. Circuits design for the electronic hardware of the signal generator.



Figure 5. The user-friendly PC interface for the operation of SG on the PC screen.

$\mathrm{Hz}$ and amplitude of $1.8 \mathrm{~V}_{\mathrm{pp}}$. The values of frequency and amplitude are determined from the horizontal and vertical scales of the SS monitor. The sampling frequency for the data acquisition is $100 \mathrm{kHz}$. The voltage scale is $0.4 \mathrm{~V}$. The observed dc offset for the triangular wave is zero. $\mathrm{CH} 2$ shows a square wave with frequency of $2.1 \mathrm{kHz}$ and amplitude of $3 \mathrm{~V}_{\mathrm{pp}}$. The period $(\Delta \mathrm{T}=$ $0.00048 \mathrm{sec}$.) and frequency $(1 / \Delta \mathrm{T}=2.1 \mathrm{e}+03 \mathrm{~Hz})$ of

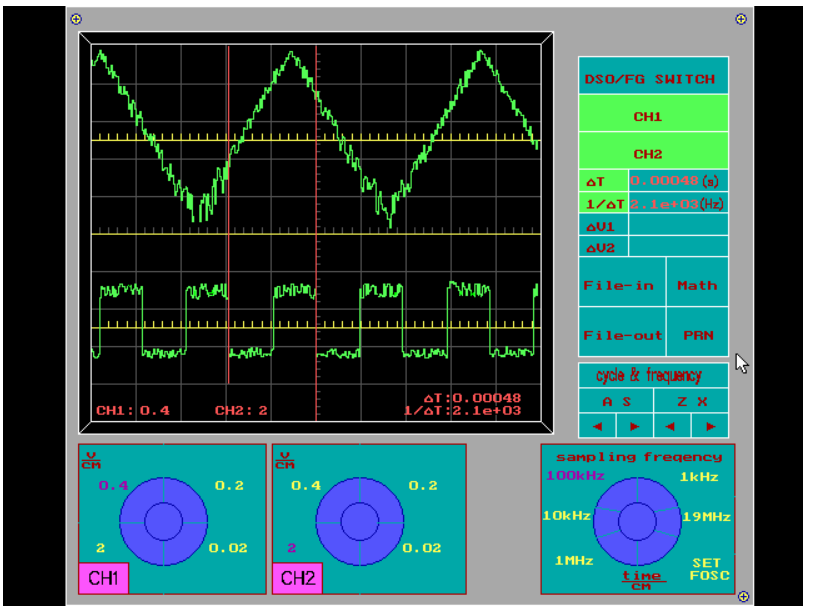

Figure 6. Experimental results of the programmable SS device. The signal sources are deduced from a $790-\mathrm{Hz}$ triangular wave of $1.8 \mathrm{~V}_{\mathrm{pp}}$ and a 2.1-kHz square wave of $3 \mathrm{~V}_{\mathrm{pp}}$.

the square wave is determined by setting a time window using two moveable straight lines displayed on the SS screen. Sampling frequencies of $1 \mathrm{k}, 10 \mathrm{k}, 100 \mathrm{k}$, and 1 $\mathrm{MHz}$ can be chosen from the SS monitor. The maximum sampling rate can be set up to $19 \mathrm{MHz}$. Figure 7 shows a square wave measured by the maximum sampling frequency of $19 \mathrm{MHz}$. The amplitude is $5 \mathrm{~V}_{\mathrm{pp}}$, duty cycle is 


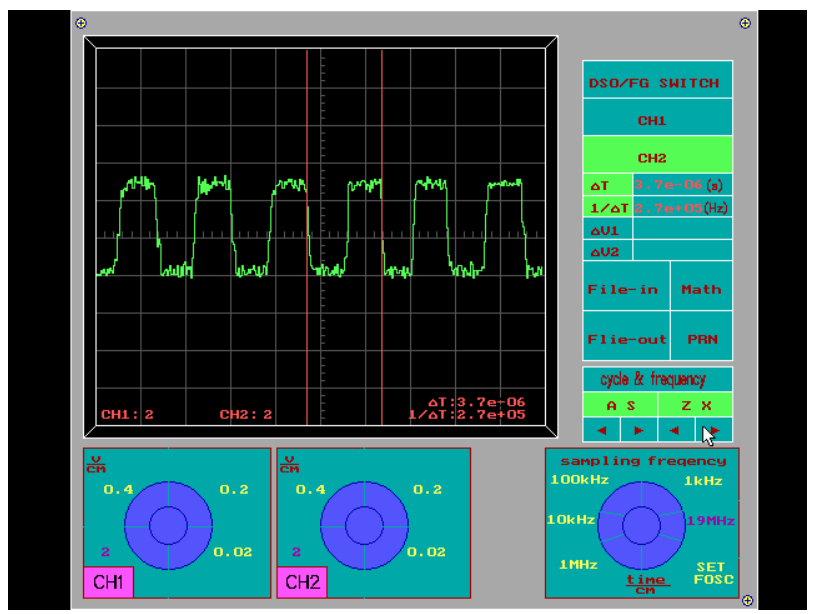

Figure 7. A square wave sampled with the maximum frequency of $19 \mathrm{MHz}$ shown on SS monitor. The signal is utilized for testing the high-frequency response of the electronic hardware of the SS card.

$50 \%$, and frequency is $270 \mathrm{kHz}$ for the square wave. The square wave shows nearly rectangular-shape waveform and which presents very low higher-order harmonic distortion in the SS monitor. This observation convinces the good performance of high-frequency response of the electronic hardware for the SS card.

To test the functional performance of the SG device, output terminal of the SG card and input terminal of the SS card is connected for each other. This configuration can simultaneously test the functional performances of both the SG and SS devices. The SG monitor shown in Figure 8(a) illustrates a user-defined signal containing a rectangular line, a semi-circle curve, and some straight lines of different slopes. The plotted waveform on the SG monitor is implemented by clicking the graphic tool keys on the left side of the SG panel, and then draws the waveform on the monitor by mouse. The amplitude of the user-defined signal is set at $4 \mathrm{~V}_{\mathrm{p}}\left(8 \mathrm{~V}_{\mathrm{pp}}\right)$ and frequency is set at $0.6 \mathrm{kHz}$. The setting values of amplitude and frequency are displayed on the right side of the SG panel in Figure 8(a). The user-defined signal is sent out to the SS device periodically via the connection of a coaxial cable. Figure 8(b) shows the measured waveform by the SS card. The signal is connected to $\mathrm{CH} 2$. The amplitude for the observed signal lies in between the voltage range of $-4 \mathrm{~V}$ to $+4 \mathrm{~V}$. From the time interval width between two straight vertical lines on the SS monitor in Figgure 8(b), a period of $\Delta \mathrm{T}=0.0017$ second (i.e. the frequency value of $600 \mathrm{~Hz}$ ) for the user-defined signal is determined. The observed amplitude and frequency are matched well with the original setting in the SG device. The observed waveform in Figure 8(b) is also the same as the user-defined curve plotted in the SG device in Figure 8(a). The experimental observations confirm the well-behaved performance for both the SG and SS de-

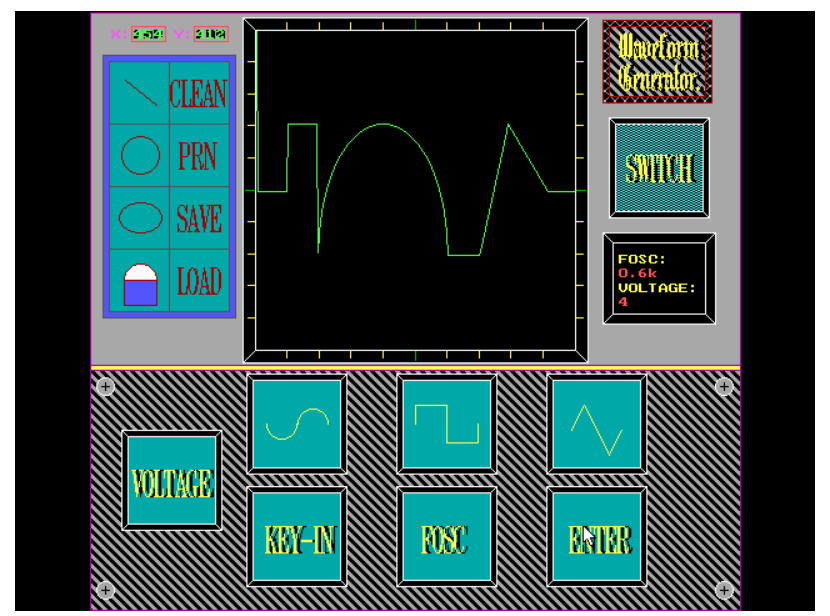

(a)

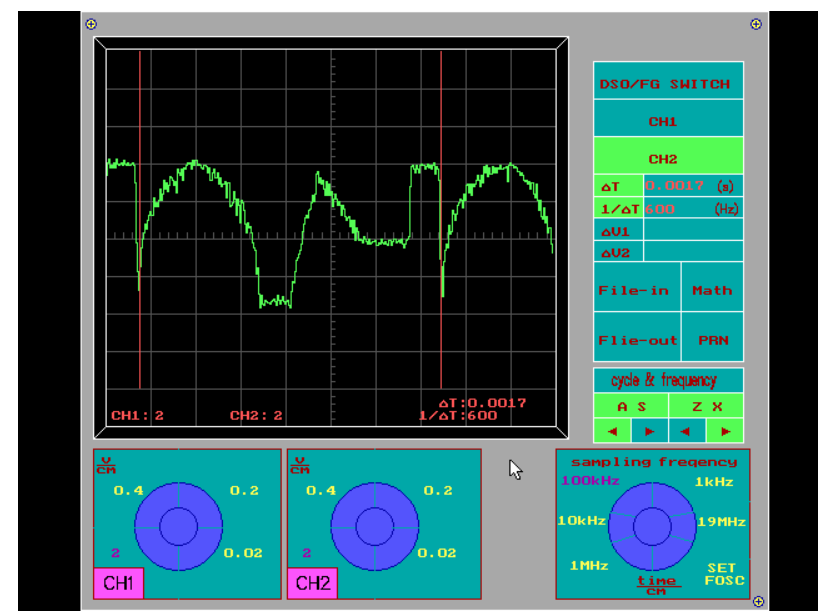

(b)

Figure 8. (a) A user-defined signal consisted of a rectangular line, a semi-circle curve and some different slopes of straight lines plotted in the SG monitor. (b) The user-defined signal observed in the SS device.

vices.

\section{Conclusion}

Design and implementation of a real instrumentation system that contains a programmable PC-based package of signals' sensing and generation are presented in this study. The hardware for both the SS and SG devices is fabricated in a plug-in style of PC I/O card so that no extra instrumentation space is needed. Design diagrams of the electronic hardware and software of the instrument are described. The good performance of high-frequency response of the SS card is verified by observing a 270$\mathrm{kHz}$ square wave with little higher-order harmonic distortion and nearly rectangular-shape waveform. To test the functional performance of the SG device, an interconnection between the output terminal of the SG card and the input terminal of the SS card is established. A 
user-defined waveform consisted of a rectangular line, a semi-circle curve and some different slopes of straight lines are used for the test. The waveform detected in the SS monitor is nearly equal to the initial design of the user-defined curve plotted in the SG monitor. This result confirms well-behaved function of the PC-based SG and SS package measurement devices. The superior functions of the package design can be summarized as follows: 1) The hardware for both the SS and SG devices is fabricated in a plug-in style of PC I/O card so that no instrumentation space is needed; 2) User-friendly operation interface that simultaneously containing both source and measurement units of the waveforms; 3) A user-defined arbitrary waveform can be easily generated using the programmable SG device.

\section{Acknowledgements}

The authors would like to acknowledge the research funding supported by the National Science Council of Taiwan under the Project No.NSC 101-2221-E-011-052MY3. Mr. Huang, M.S. is much appreciated for technical assistance to this work.

\section{REFERENCES}

[1] P. Pellati and P. Olivo, "Automated Test Equipment for Research on Nonvolatile Memories," IEEE Transactions on Instrumentation and Measurement, Vol. 50, No. 5, 2001, pp. 1162-1166. doi:10.1109/19.963177
[2] P. Bilski and W. Winiecki, "Virtual Spectrum Analyzer Based on Data Acquisition Card," IEEE Transactions on Instrumentation and Measurement, Vol. 51, No. 1, 2002, pp. 82-87. doi:10.1109/19.989906

[3] L. F. F. B. Palma and A. R. F. da Silva, "A Virtual Instrumentation Support System," IEEE International Conference on Electronics, Circuits and Systems, Vol. 3, Potugal, 7-10 September 1998, pp. 273-276.

[4] G. Cauffet and J.-P. Keradec, "Digital Oscilloscope Measurements in High-Frequency Switching Power Electronics," IEEE Transactions on Instrumentation and Measurement, Vol. 41, No. 6, 1992, pp. 856-860. doi:10.1109/19.199422

[5] C. H. Ho, "A Practical and Inexpensive Design for Measuring the Radiation Patterns and Luminescent Spectra of Optoelectronic Devices," Review of Scientific Instruments, Vol. 72, No. 7, 2001, pp. 3103-3107. doi:10.1063/1.1379601

[6] C. H. Ho and H. W. Lee, "High-Resolution and Easily Implemented Spectral Measured System Used for Optical Characterization of Optoelectronic Materials and Devices," Optical Engineering, Vol. 43, No. 7, 2004, pp. 1628-1633. doi:10.1117/1.1755718

[7] Analog Device Corp., "Amplifier Reference Manual," Analog Device Corp., Norwood, 1992, pp. 363-374.

[8] Analog Device Corp., "Data Converter Reference Manual," vol. II, Analog Device Corp., Norwood, 1992, pp. 781788. 